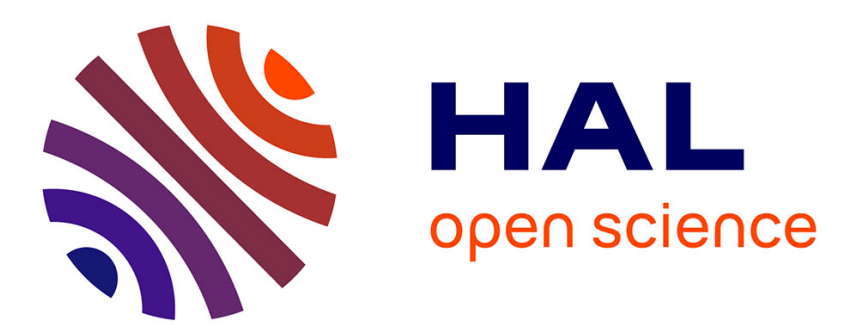

\title{
Anisotropy of the Sommerfeld Coefficient in Magnesium Diboride Single Crystals
}

Z. Pribulova, Thierry Klein, Jacques Marcus, C. Marcenat, F. Levy-Bertrand, M. S. Park, H. G. Lee, B.W. Kang, S. I. Lee, S. Tajima, et al.

\section{- To cite this version:}

Z. Pribulova, Thierry Klein, Jacques Marcus, C. Marcenat, F. Levy-Bertrand, et al.. Anisotropy of the Sommerfeld Coefficient in Magnesium Diboride Single Crystals. Physical Review Letters, 2007, 98 (13), pp.137001. 10.1103/PhysRevLett.98.137001 . hal-00957175

HAL Id: hal-00957175

https://hal.science/hal-00957175

Submitted on 10 Mar 2014

HAL is a multi-disciplinary open access archive for the deposit and dissemination of scientific research documents, whether they are published or not. The documents may come from teaching and research institutions in France or abroad, or from public or private research centers.
L'archive ouverte pluridisciplinaire HAL, est destinée au dépôt et à la diffusion de documents scientifiques de niveau recherche, publiés ou non, émanant des établissements d'enseignement et de recherche français ou étrangers, des laboratoires publics ou privés. 


\title{
Anisotropy of the Sommerfeld Coefficient in Magnesium Diboride Single Crystals
}

\author{
Z. Pribulova, ${ }^{1,2, *}$ T. Klein,,${ }^{1,2}$ J. Marcus, ${ }^{1}$ C. Marcenat, ${ }^{3}$ F. Levy, ${ }^{3}$ M. S. Park, ${ }^{4}$ H. G. Lee, ${ }^{4}$ B. W. Kang, ${ }^{4}$ S. I. Lee, ${ }^{4}$ \\ S. Tajima, ${ }^{5}$ and S. Lee ${ }^{6}$ \\ ${ }^{1}$ Institut Néel-CNRS, F-38042 Grenoble Cedex 9, France \\ ${ }^{2}$ Université Joseph Fourier, F-38041 Grenoble, France \\ ${ }^{3}$ CEA-Grenoble, Département de Recherche Fondamentale sur la Matière Condensée, F-38054 Grenoble, France \\ ${ }^{4}$ NVCRICS and Department of Physics, Pohang University, Pohang 790-784 Republic of Korea \\ ${ }^{5}$ Department of Physics, Osaka University, Osaka 560-0043, Japan \\ ${ }^{6}$ Superconductivity Research Laboratory, ISTEC, Shinonome 1-10-13, Koto-ku, Tokyo 135-0062, Japan
}

(Received 21 December 2006; published 28 March 2007)

\begin{abstract}
The anisotropic field dependence of the Sommerfeld coefficient $\gamma$ has been measured down to $B \rightarrow 0$ by combining specific heat and Hall probe magnetization measurements in $\mathrm{MgB}_{2}$ single crystals. We find that $\gamma(B, \theta)$ is the sum of two contributions arising from the $\sigma$ and $\pi$ band, respectively. We show that $\gamma_{\sigma}(B, \theta)=B / B_{c 2}(\theta)$ where $B_{c 2}(\theta)=B_{c 2}^{\mathrm{ab}} / \sqrt{\sin ^{2} \theta+\Gamma^{2} \cos ^{2} \theta}$ with $\Gamma \sim 5.4(\theta$ being the angle between the applied field and the $c$ axis) and $\gamma_{\pi}(B, \theta)=\gamma_{\pi}(B)=B / B_{\pi}(B)$. The "critical field" of the $\pi$ band $B_{\pi}$ is fully isotropic but field dependent increasing from $\sim 0.25 \mathrm{~T}$ for $B \leq 0.1 \mathrm{~T}$ up to $3 \mathrm{~T} \sim B_{c 2}^{c}$ for $B \rightarrow 3 \mathrm{~T}$. Because of the coupling of the two bands, superconductivity survives in the $\pi$ band up to $3 \mathrm{~T}$ but is totally destroyed above for any orientation of the field.
\end{abstract}

DOI: 10.1103/PhysRevLett.98.137001

It is now well established that the anisotropy parameter $(\Gamma)$ of magnesium diboride $\left(\mathrm{MgB}_{2}\right)$ is strongly field and temperature dependent $[1,2]$. This is a direct consequence of the coexistence of two weakly coupled superconducting bands. As suggested by point contact spectroscopy [3] or small angle neutron scattering [4] experiments, the socalled $\pi$ band is very sensitive to magnetic field and, above some "crossover" field on the order of $0.5-1 \mathrm{~T}$, the anisotropy is then mainly given by the parameters of the quasi2D $\sigma$ band leading to $\Gamma=\Gamma_{H_{c 2}}=H_{c 2}^{\mathrm{ab}} / H_{c 2}^{c}(\sim 5-6$ at low temperature, $H_{c 2}^{\mathrm{ab}}$ and $H_{c 2}^{c}$ being the upper critical fields parallel to the $a b$ planes and $c$ axis, respectively). On the other hand, at low field, the anisotropy has to be averaged over the entire Fermi surface [5] leading to $\Gamma \sim \Gamma_{H_{c 1}} \sim 1$ in good agreement with $H_{c 1}$ measurements [2].

Similarly, as a consequence of this suppression of the $\pi$ band, preliminary measurements of the Sommerfeld coefficient $\gamma=\lim C_{\mathrm{el}} /\left.T\right|_{T \rightarrow 0}\left(C_{\mathrm{el}}\right.$ being the electronic contribution, to the specific heat) by Bouquet et al. [6] clearly showed that its field dependence is highly nonlinear. However, the details of the nature of the superconducting state remained unknown. We will show here that, above $\sim 0.3 \mathrm{~T}$, superconductivity is induced in the $\pi$ band by coupling with the $\sigma$ band leading to a shrinking of the vortex core from $\xi_{\pi}(0) \sim 350 \AA$ down to $\xi_{\pi}=$ $\xi_{\sigma}^{c} \sim 100 \AA$ for $B \sim 3 \mathrm{~T}$. Superconductivity is completely destroyed in this band above $3 \mathrm{~T}$ for any orientation of the magnetic field.

We present the first detailed analysis of the angular and field dependence of $\gamma$ by combining specific heat and Hall probe magnetization measurements. In classical superconductors, the angular dependence of $\gamma$ is determined by the $B / B_{c 2}$ ratio with $B_{c 2}=B_{c 2}^{\mathrm{ab}} / \sqrt{\sin ^{2} \theta+\Gamma^{2} \cos ^{2} \theta}$ ( $\theta$ being the angle between the $c$ axis and the field). We show that, in
PACS numbers: 74.70.Ad, 74.25.Op, 74.72.- $\mathrm{h}$

$\mathrm{MgB}_{2}$, the contribution of the $\sigma$ band to $\gamma$ is directly proportional to $B / B_{c 2}(\theta)$, whereas the contribution of the $\pi$ band is isotropic but highly nonlinear in field. For $T \geq$ $10 \mathrm{~K}$, the influence of the small gap is smeared out by the temperature and $\Delta C_{p} / T=f\left(B / B_{c 2}(T, \theta)\right)$. Specific heat measurements have been performed on single crystals of $\mathrm{MgB}_{2}$ grown under high pressure [7,8] (with typical dimensions of few hundred microns) using an ac technique. This high sensitivity technique is very well adapted to measure $C_{p}$ of very small samples and to carry continuous measurements during field sweeps at a given temperature. We were thus able to obtain the field dependence of the Sommerfeld coefficient continuously on the entire field range for different $\theta$ values. A precise in situ calibration of the thermocouple used to record the temperature oscillations was obtained from measurements on ultrapure silicon.

Figure 1 displays this field dependence for $H \| c$ and $H \|$ ab (at $T=2 \mathrm{~K}$ ). As previously observed by Bouquet et al., the $\gamma$ vs $H_{a}$ curve is nonlinear. Those measurements suggested that $\gamma$ is isotropic below $0.2 \mathrm{~T}$, becoming anisotropic for $H \geq 0.5 \mathrm{~T}$. However, for fields up to a few $H_{p}$, the first penetration field, the measurements are strongly hysteretic reflecting different vortex distributions in the sample [see [9] and inset of Fig. 1(a)]. The proximity of $H_{p}$ may thus cast some doubt on the field dependence of $\gamma$ observed in [6] at low field. As shown in the inset of Fig. 1(a), the first penetration field $H_{p}$ can be clearly identified on the ascending branch of zero field cooled cycles as $\gamma=0$ up to $H_{a}=H_{p}$ and rises sharply above this field due to the fast proliferation of vortices in the sample. Vortices remain pinned in the samples for decreasing fields and $\gamma \neq 0$ down to $H_{a}=0$. To clearly identify the field dependence of $\gamma$ in this region it was important to 

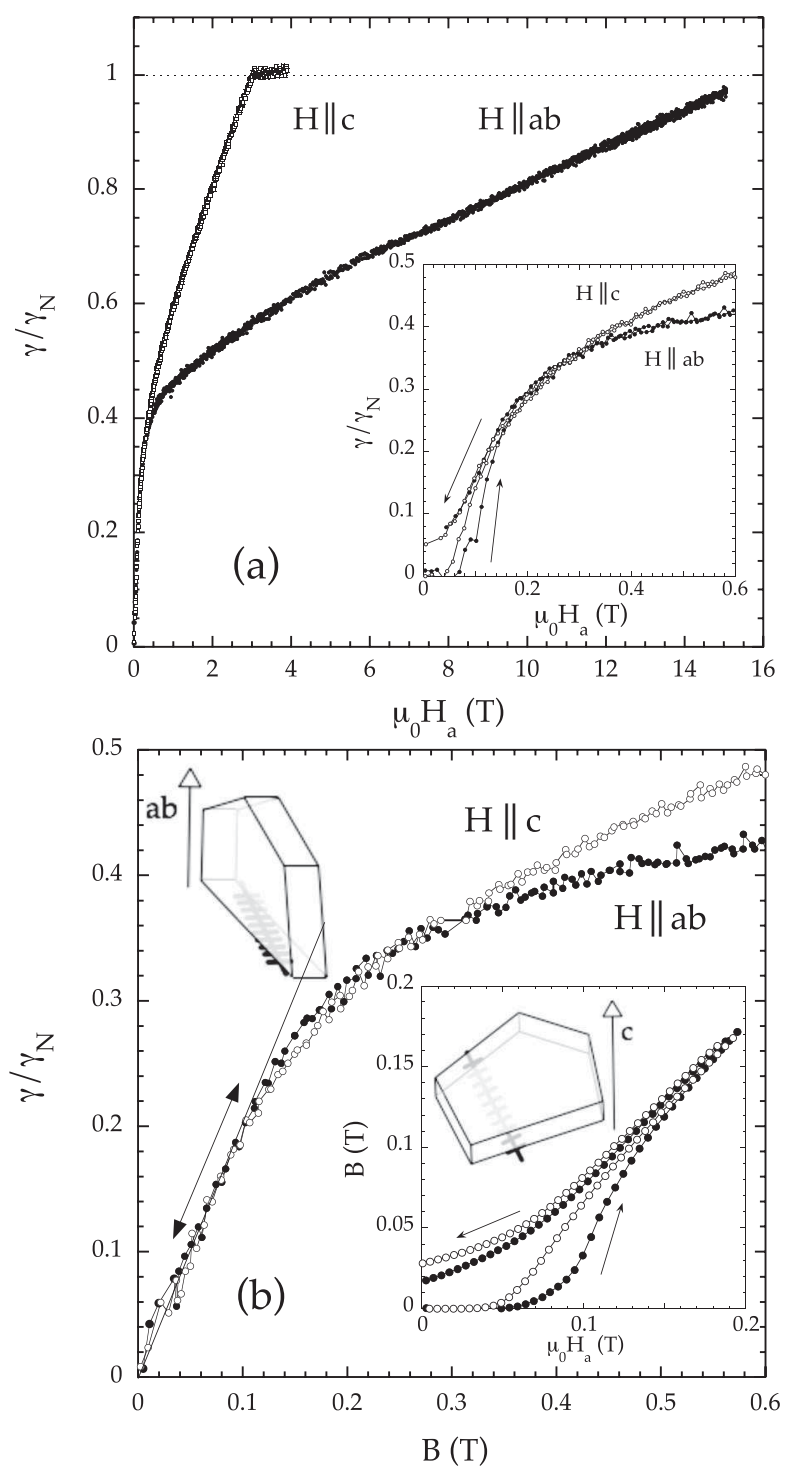

FIG. 1. Magnetic field dependence of the Sommerfeld coefficient $\gamma$ at $T=2.5 \mathrm{~K}$ for $H \| c$ and $H \|$ ab. In the inset: low field dependence showing the hysteretic behavior related to flux penetration and pinning. (b) Sommerfeld coefficient $\gamma$ for $H \| c$ and $H \|$ ab as a function of the average field $B$. In the inset: average field $B$ deduced by Hall probe magnetometry (see sketches) as a function of the applied field $H_{a}$.

determine the true induction $B$ in the sample. We thus performed Hall probe magnetization measurements on the same sample for both field directions using a miniature Hall probe array [see sketches in Fig. 1(b)]. The induction $B$ at the surface of the sample has been averaged over $\sim 25$ points for $H \| c$ and $\sim 10$ points for $H \|$ ab (the average field is hereafter noted $B$ ). The $B$ vs $H_{a}$ curves are displayed in the inset of Fig. 1(b) and the corresponding $\gamma$ vs $B$ curves in Fig. 1(b). As shown, $\gamma$ is perfectly linear and isotropic at low field (note that, as expected $\gamma$ vs $B$ is completely reversible). However, the linear regime is only visible up to $\sim 0.1 \mathrm{~T}$ and $\gamma$ remains isotropic up to $B \sim 0.3 \mathrm{~T}$.
As discussed in [6], in $\mathrm{MgB}_{2}$ the nonlinear behavior can be qualitatively understood by writing $\gamma=\omega \gamma_{\pi}+(1-$ $\omega) \gamma_{\sigma}$, where $\omega$ is the relative weight of the $\pi$ band (on the order of $\frac{1}{2}$ [10]). Assuming that all excitations are localized in the vortex cores, i.e., that $\gamma_{i} \propto \gamma_{N}\left(\xi_{i} / a_{0}\right)^{2}$ for $B<B_{i}=$ $\Phi_{0} / 2 \pi \xi_{i}^{2}$ and $\gamma_{i}=\gamma_{N}$ for $B>B_{i}$ (with $i=\pi$ or $\sigma$ ), one gets two linear behaviors corresponding to $B<B_{\pi}$ and $B>B_{\pi}$, respectively [introducing $a_{0} \sim \sqrt{\left(\Phi_{0} / B\right)}$ ]. The low field linear behavior is hence clearly visible but limited on a very restricted field range and, as discussed below, the high field linear behavior is only observed for $\theta \neq 0$ (for $B>3 \mathrm{~T}$ ).

To get a better description of $\gamma(B, \theta)$, we measured the angular dependence of the Sommerfeld coefficient for various applied fields [see inset of Fig. 2(a), at $T=2 \mathrm{~K}$ ]. In a classical single gap superconductor $\gamma$ is fully determined by the $B / B_{c 2}$ ratio. In clean systems, deviations from the above mentioned linear behavior may be expected [11], but $\gamma$ still remains a function of $B / B_{c 2}$. Obviously such a simple behavior does not hold in $\mathrm{MgB}_{2}$ since $\gamma$ is isotropic up to $\approx 0.3 \mathrm{~T}$ and its anisotropy then rises up to 5-6 for $B \rightarrow B_{c 2}$. However, as shown in Fig. 2(a), substracting from $\gamma(\theta)$ a constant for each $H_{a}$ value, i.e., taking $\left[\gamma / \gamma_{N}-\omega a(B)\right] /(1-\omega)$ instead of $\gamma / \gamma_{N}$, leads to a collapse of all the data on a single curve when plotted as a function of $B / B_{c 2}(\theta) \propto B \sqrt{\sin ^{2} \theta+\Gamma^{2} \cos ^{2} \theta}$ [12] with $\Gamma=\Gamma_{H_{c 2}}=5.4$. We here assumed that $\gamma_{\pi}(B, \theta)$ is isotropic and hence depends only on $B\left[\gamma_{\pi} / \gamma_{N}=a(B)\right]$ and that $\gamma_{\sigma}(B, \theta) / \gamma_{N}=\left[\gamma / \gamma_{N}-\omega a(B)\right] /(1-\omega)=$ $f\left(B / B_{c 2}(\theta)\right)$. It is important to note that we made no assumption on the form of the function $f$ and directly got $\gamma_{\sigma}(B, \theta)=\left[B / B_{c 2}(\theta)\right] \gamma_{N}$ as expected in classical (dirty) superconductors. Note also that $\gamma$ appears to be fully isotropic below $0.3 \mathrm{~T}$ but, in this field range, the contribution of the $\sigma$ band is less than a few percent and the corresponding variation is at the limit of our experimental resolution [13].

The $a(B)=\gamma_{\pi} / \gamma_{N}$ values are displayed in the inset of Fig. 2(b) (solid symbols) together with direct determinations from magnetic field sweeps for the indicated $\theta$ values: $\quad \gamma_{\pi} / \gamma_{N}=\left[\gamma / \gamma_{N}-(1-\omega) B / B_{c 2}(\theta)\right] / \omega \quad$ (open symbols). For $B \geq 3 \mathrm{~T}, \gamma_{\pi}=\gamma_{N}$ showing that superconductivity is completely destroyed in this band at high field. This is further emphasized in the field dependence of $\gamma$ at fixed $\theta \neq 0$ values [Fig. 2(b)] which clearly shows that $\gamma$ becomes perfectly linear for $B \geq 3 \mathrm{~T}$ in all directions. Note that the linear fits intercept the $B=0$ axis at $\omega \sim$ 0.5 in good agreement with numerical calculations [10]. With this $\omega$ value, we did not observe any linear high field behavior for $H \| c$ but $\gamma$ can be rather described by a $\left(B / B_{c 2}^{c}\right)^{\alpha}$ law (with $\alpha \sim 0.4-0.5$ ). As discussed in [14], it is then possible to introduce an effective field dependent $\xi_{\text {eff }} \quad$ value: $\quad \xi_{\text {eff }}(B)=\sqrt{\omega \xi_{\pi}(B)^{2}+(1-\omega) \xi_{\sigma}^{2}} \quad$ with $a(B)=\left[\xi_{\pi}(B) / a_{0}\right]^{2}$ (for $B \leq 3 \mathrm{~T}$ ). This effective value, combined with a field dependent penetration depth can then be used to described all physical properties (see also 

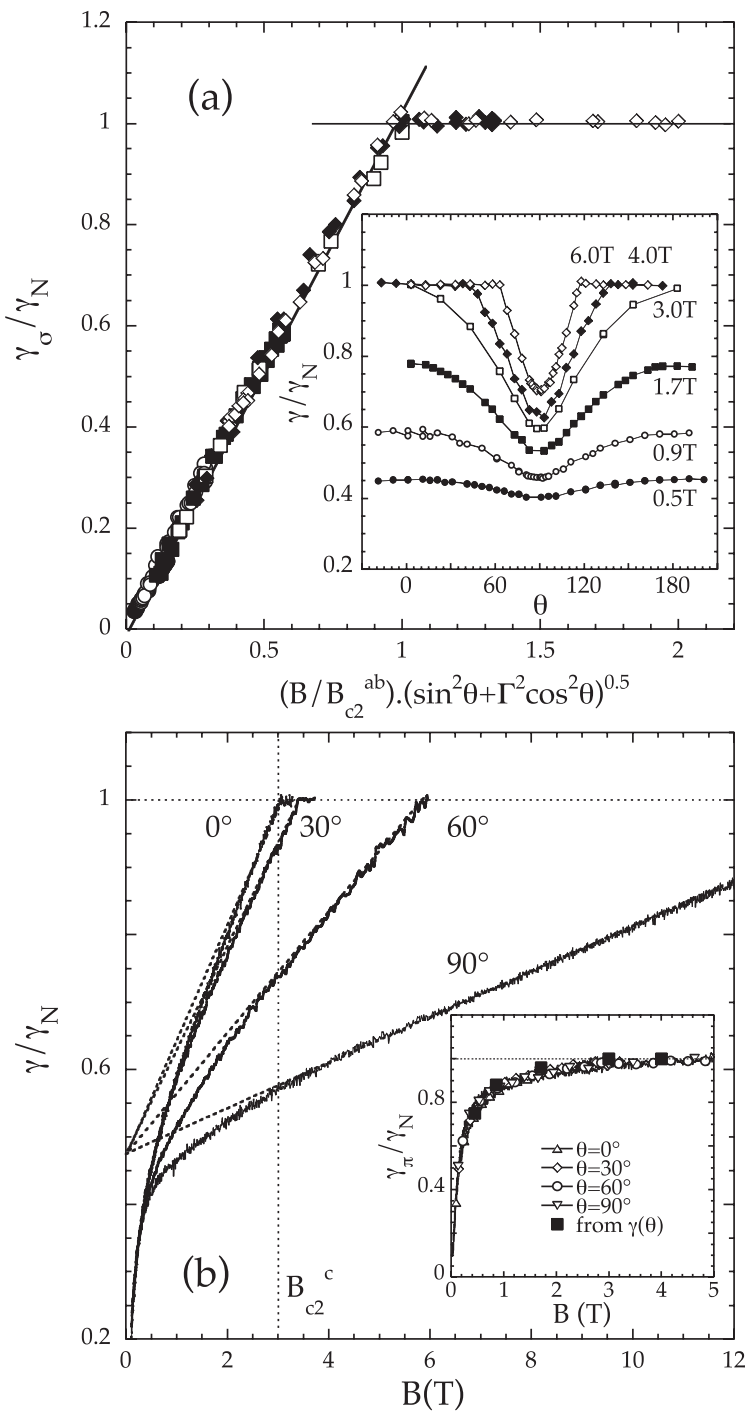

FIG. 2. (a) $\left[\gamma / \gamma_{N}-\omega a(B)\right] /(1-\omega)$ as a function of $B / B_{c 2}$ with $B_{c 2}=B_{c 2}^{a b} / \sqrt{\sin ^{2} \theta+\Gamma^{2} \cos ^{2} \theta}$ (with $\omega \sim \frac{1}{2}$ and $\Gamma \sim 5.4$ ), in the inset $\gamma / \gamma_{N}$ as a function of $\theta$ for the indicated values of the applied field. (b) Field dependence of the Sommerfeld coefficient for the indicated field directions showing that $\gamma$ becomes linear for $B \geq 3 \mathrm{~T}$. In the inset: field dependence of the contribution of the $\pi$ band: $\gamma_{\pi} / \gamma_{N}=a(B)$ deduced from the angular measurements (solid squares) and magnetic sweeps at the indicated angles (open symbols, see text for details).

[15]). The $\xi_{\pi}(0)$ value would correspond to a critical field for the $\pi$ band on the order of $B_{\pi}(0)=0.25 \mathrm{~T}$ but superconductivity still survives in this band due to the coupling with the $\sigma$ band leading to a shrinking of the vortex core from $\xi_{\pi} \approx 350 \AA$ below $0.1 \mathrm{~T}$ down to $\xi_{\pi}=\xi_{\sigma}^{\text {ab }}$ for $B \sim$ $3 \mathrm{~T}=B_{c 2}^{c}$. However, it is important to note that, since the $\pi$ band is isotropic, it can have only one $\theta$ independent $B_{c 2}$ value and superconductivity is hence destroyed in this band in all direction for $B \geq 3 \mathrm{~T}$.

As expected the $\xi_{\sigma}^{\mathrm{ab}} \sim 100 \AA$ and $\xi_{\sigma}^{c} \sim 20 \AA$ values are very close to the BCS single band estimate: $\hbar v_{F, \sigma} / \pi \Delta_{\sigma} \sim$ $130 \AA$ and $\sim 20 \AA$ in the $a b$ planes and along the $c$ direc- tion, respectively $\left(v_{F, \sigma}\right.$ being the Fermi velocity of the $\sigma$ band $\sim 4.6 \times 10^{7} \mathrm{~cm} / \mathrm{s}$ and $\sim 0.7 \times 10^{7} \mathrm{~cm} / \mathrm{s}$ for the two crystallographic directions and $\Delta_{\sigma}$ the large gap value $\sim 7.0 \mathrm{meV})$. More surprisingly, the as-deduced $\xi_{\pi}$ value $(\sim 350 \AA)$ is also quite close to $\hbar v_{F, \pi} / \pi \Delta_{\pi} \sim 400-500 \AA$ [taking an average $v_{F, \pi}$ value on the order of (5-6) $\times$ $10^{7} \mathrm{~m} / \mathrm{s}[5,16]$ and $\left.\Delta_{\pi} \sim 2.4 \mathrm{meV}\right]$. Indeed, it has been suggested by Zhitomirsky et al. [16] that this single band estimate should not be applicable in $\mathrm{MgB}_{2}$ and that in the clean limit $\xi_{\pi}^{c} / \xi_{\sigma}^{c} \leq \sqrt{\omega\left\langle v_{F, \pi}^{2}\right\rangle /(1-\omega)\left\langle v_{F, \sigma}^{2}\right\rangle} \sim 1-2$ for reasonable $\left\langle v_{F, \pi}^{2}\right\rangle /\left\langle v_{F, \sigma}^{2}\right\rangle$ values [5,16]. Similarly, it has been shown that, in the dirty limit, $\xi_{\pi}^{c} / \xi_{\sigma}^{c} \sim 3$ for $D_{\pi} / D_{\sigma} \sim 4$ [17] (where $D_{i}$ is the diffusivity of the $i$ band), i.e., for a $\sigma$ band which would be much dirtier than the $\pi$ band. This numerical $\xi_{\pi}$ value obviously deserves further theoretical investigation.

As discussed in [6], an effective anisotropy ratio can be defined as the ratio of the magnetic fields in the $a b$ plane and $c$ axis which correspond to the same $\gamma$ value but, as pointed out in [6], the choice of the corresponding field is then arbitrary and an almost linear increase of $\Gamma$ with field was proposed. However, we have seen that superconductivity is completely destroyed in the $\pi$ band for $B \geq 3 \mathrm{~T}$ and $\Gamma$ is hence expected to be equal to 5.4 above $3 \mathrm{~T}$. We have calculated this field dependent anisotropy $\Gamma(B)$, writing $\gamma(B, \theta)=\gamma\left(B \sqrt{\sin ^{2} \theta+\Gamma(B)^{2} \cos ^{2} \theta}, \theta=90^{\circ}\right)$, i.e., introducing an effective field dependent $B_{c 2}^{*}=$ $B_{c 2}^{\mathrm{ab}} / \sqrt{\sin ^{2} \theta+\Gamma(B)^{2} \cos ^{2} \theta}$ and writing that $\gamma=$ $f\left(B / B_{c 2}^{*}\right)$. The corresponding $\Gamma(B)$ values have been reported on Fig. 3 (open symbols and lines) for $\theta=0^{\circ}, 30^{\circ}$, and $60^{\circ}$ together with the $\Gamma_{\lambda}=\frac{\lambda_{c}}{\lambda_{\mathrm{ab}}}$ values deduced from

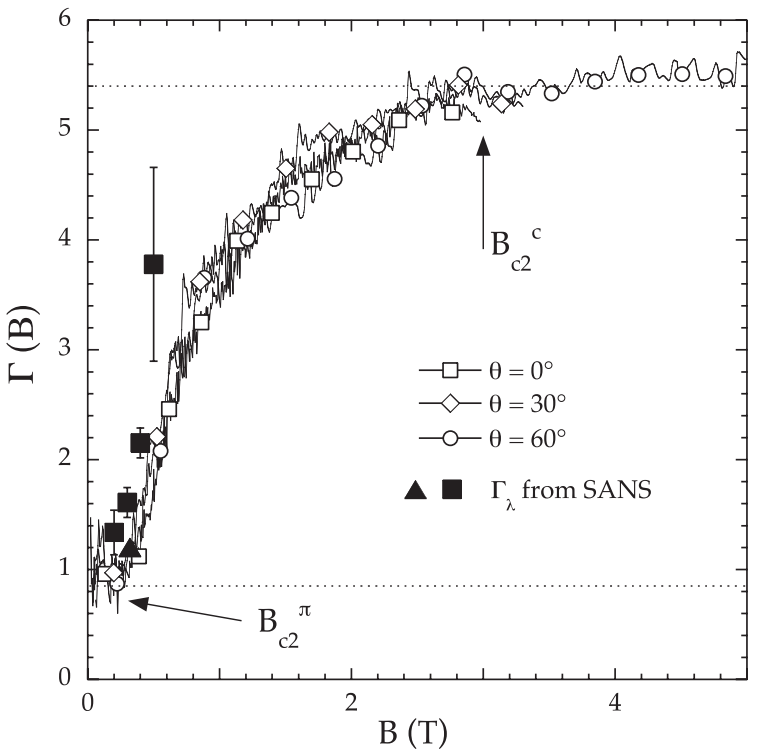

FIG. 3. Field dependence of the anisotropy ratio (see text for details) together with $\Gamma_{\lambda}=\frac{\lambda_{c}}{\lambda_{\mathrm{ab}}}$ values deduced from small angle neutron scattering data (closed squares: from [4], closed triangle: from [16]. 


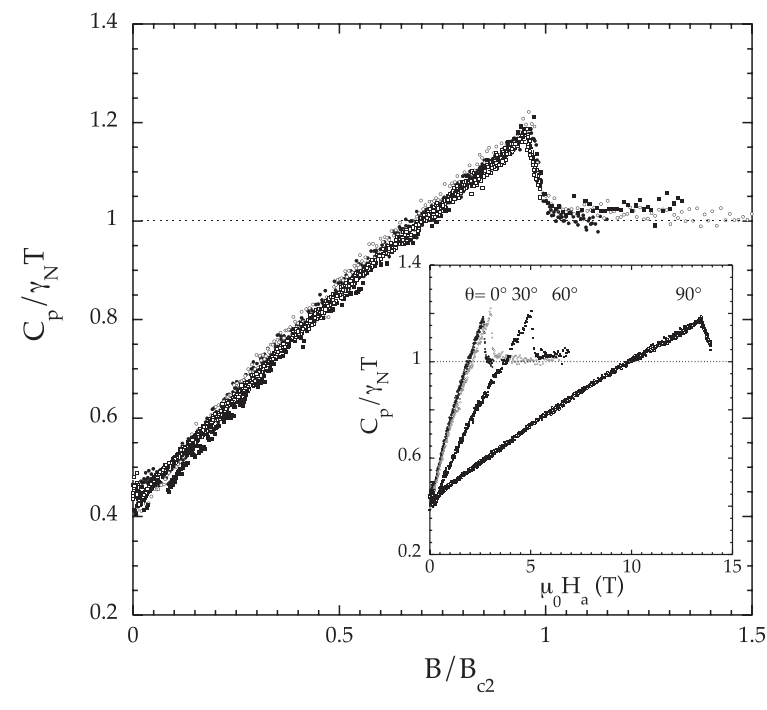

FIG. 4. (a) Magnetic field dependence of the specific heat at $T=9 \mathrm{~K}$ showing that, at high temperature, $C_{p} / T$ scales as $B / B_{c 2}(\theta)$. In the inset: same dependence plotted as a function of the applied field $H_{a}$ for $\theta=0^{\circ}, 30^{\circ}, 60^{\circ}$, and $90^{\circ}$.

small angle neutron scattering data (closed squares: from [4], closed triangle: from [18]). As shown the same effective anisotropy (saturating at 5.4 for $B \geq 3 \mathrm{~T}$ ) is obtained for all angles and a very reasonable agreement is obtained between specific heat and SANS data confirming that $\Gamma_{\lambda}=$ $\Gamma=\Gamma_{\xi}$. Note that $\Gamma$ stays on the order of 1 up to $B_{\pi}$ and then sharply increases reaching $\Gamma_{H_{c 2}}$ for $B \geq B_{c 2}^{c}$.

Finally, we have investigated the influence of the temperature on the field and angular dependence of $C_{p}$. The inset of Fig. 4 displays the field dependence of $C_{p} / T$ at $T=9 \mathrm{~K}$ for the indicated $\theta$ values. In this temperature range superconductivity in the $\pi$ band is reduced due to thermal activation over the small gap and, as shown in the main panel of Fig. 4, all curves can then be rescaled directly as a function of $B / B_{c 2}(\theta)$ clearly showing that a classical behavior is recovered at high temperature (except that $\Gamma$ is temperature dependent).

We have shown that, the contribution of the $\sigma$ band to the specific heat $\gamma_{\sigma}(B, \theta)=\left[B / B_{c 2}(\theta)\right] \gamma_{N}$ whereas the contribution of the $\pi$ band is isotropic but highly nonlinear in field: $\gamma_{\pi}(B, \theta)=\gamma_{\pi}(B)=\left[B / B_{\pi}(B)\right] \gamma_{N}$ for $B \leq$ $3 \mathrm{~T} \sim B_{c 2}^{c}$ and $\gamma_{\pi}=\gamma_{N}$ for $B \geq 3 \mathrm{~T}$. We hence show that superconductivity can be induced in the $\pi$ band by coupling with the $\sigma$ band but only up to $B_{c 2}^{c}$ and no superconductivity is observed in this band for $B \geq 3 \mathrm{~T}$.

We would like to thank M. Konczykowski and V. Mosser for their help in the Hall probe magnetization measurements. Z.P. thanks the Slovak Science and Technology Agency for partial support, Contracts No. APVT-51016604 and No. LPP-0101-06. S. L. thanks the New Energy and Industrial Technology Development Organization (NEDO) for partial support.
*Present address: Centre of Low Temperature Physics IEP SAS \& FS UPJS, Watsonova 47, SK-04001 Kosice, Slovakia.

[1] L. Lyard et al., Phys. Rev. B 66, 180502(R) (2002); S. L. Bud'ko, V. G. Kogan, and P. C. Canfield, Phys. Rev. B 64, 180506 (2001); M. Angst et al., Phys. Rev. Lett. 88, 167004 (2002); U. Welp et al., Phys. Rev. B 67, 012505 (2003).

[2] L. Lyard, P. Szabo, T. Klein, J. Marcus, C. Marcenat, K. H. Kim, B. W. Kang, H. S. Lee, and S. I. Lee, Phys. Rev. Lett. 92, 057001 (2004).

[3] P. Szabó et al., Phys. Rev. Lett. 87, 137005 (2001); P. Samuely et al., Physica (Amsterdam) 385C, 244 (2003).

[4] R. Cubitt, M. R. Eskildsen, C. D. Dewhurst, J. Jun, S. M. Kazakov, and J. Karpinski, Phys. Rev. Lett. 91, 047002 (2003).

[5] V. G. Kogan and S.L. Bud'ko, Physica (Amsterdam) 385C, 131 (2003).

[6] F. Bouquet, Y. Wang, L. Sheitkin, T. Plackowski, A. Junod, S. Lee, and S. Tajima, Phys. Rev. Lett. 89, 257001 (2002).

[7] S. Lee, H. Mori, T. Masui, Y. Eltsev, A. Yamamoto, and S. Tajima, J. Phys. Soc. Jpn. 70, 2255 (2001).

[8] C. U. Jung, J. Y. Kim, P. Chowdhury, Kijoon H. P. Kim, Sung-Ik Lee, D. S. Koh, N. Tamura, W. A. Caldwell, and J. R. Patel, Phys. Rev. B 66, 184519 (2002).

[9] L. Lyard, T. Klein, J. Marcus, R. Brusetti, C. Marcenat, M. Konczykowski, V. Mosser, K. H. Kim, B. W. Kang, H. S. Lee, and S.I. Lee, Phys. Rev. B 70, 180504(R) (2004).

[10] A. Y. Liu, I. I. Mazin, and J. Kortus, Phys. Rev. Lett. 87, 087005 (2001); A. A. Golubov et al., J. Phys. Condens. Matter 14, 1353 (2002); H. J. Choi et al., Nature (London) 418, 758 (2002); I. I. Mazin et al., Phys. Rev. Lett. 89, 107002 (2002).

[11] V. G. Kogan, Phys. Rev. B 66, 020509(R) (2002); V. G. Kogan and N. V. Zhelezina, Phys. Rev. B 69, 132506 (2004).

[12] Small deviations from the $B_{c 2}=B_{c 2}^{\mathrm{ab}} / \sqrt{\sin ^{2} \theta+\Gamma^{2} \cos ^{2} \theta}$ law may be expected in two gap superconductors but those deviations are extremely small at low temperature: A. Rydh, U. Welp, A. E. Koshelev, W. K. Kwok, G. W. Crabtree, R. Brusetti, L. Lyard, T. Klein, C. Marcenat, B. Kang, K. H. Kim, K. H. P. Kim, H.-S. Lee, and S.-I. Lee, Phys. Rev. B 70, 132503 (2004).

[13] The anistropy of the $\sigma$ band might also be compensated by a small anisotropy of the $\pi$ band $[5,16]$.

[14] T. Klein, L. Lyard, J. Marcus, Z. Holanova, and C. Marcenat, Phys. Rev. B 73, 184513 (2006).

[15] M. Eisterer, M. Zehetmayer, H. W. Weber, and J. Karpinski, Phys. Rev. B 72, 134525 (2005).

[16] M.E. Zhitomirsky and V.-H. Dao, Phys. Rev. B 69, 054508 (2004).

[17] A. E. Koshelev and A. A. Golubov, Phys. Rev. Lett. 90, 177002 (2003).

[18] D. Pal, L. DeBeer-Schmitt, T. Bera, R. Cubitt, C. D. Dewhurst, J. Jun, N. D. Zhigadlo, J. Karpinski, V. G. Kogan, and M. R. Eskildsen, Phys. Rev. B 73, 012513 (2006). 